\title{
An optimization and visualization method of cutting lines for lettering images clipped from adhesive tape (Withdrawal Notice)
}

Hiroki Mori, Kunio Sakamoto

Hiroki Mori, Kunio Sakamoto, "An optimization and visualization method of cutting lines for lettering images clipped from adhesive tape (Withdrawal Notice)," Proc. SPIE 10817, Optoelectronic Imaging and Multimedia Technology V, 1081700 (2 November 2018); doi: 10.1117/12.2327075 


\section{An optimization and visualization method of cutting lines for lettering images clipped from adhesive tape (Withdrawal Notice)}

Hiroki Mori, and Kunio Sakamoto

Konan Univ. (Japan)

Proc. SPIE 10817, 1081700 (2018)

Online Publication Date: 2 November 2018

Withdrawn from Publication: 4 February 2019

Conference Date: 11 October-12 October 2018

Conference Location: Beijing, China

Conference Title: Optoelectronic Imaging and Multimedia Technology $\mathrm{V}$

Conference Chairs: Qionghai Dai, Tsutomu Shimura

Publisher's Note: This manuscript, originally published on 2 November 2018, has been withdrawn by the publisher for editorial reasons. 